\title{
Application of Discovery Teaching Method in the Teaching of Density Function
}

\author{
Pin Wang \\ Guangzhou College of Commerce, Guangzhou 511363, China. \\ 1040168586@qq.com
}

\begin{abstract}
Taking density function of continuous random variables in probability statistics as an example, this paper analyzes the learning mode and psychological mechanism of mathematical concepts in undergraduate business schools based on Bruner's discovery teaching theory. This paper discusses the process and approach of the freshman major in economics from "passive learning" to "active learning" and adopts the five-step method of discovery teaching method: creating situation $\rightarrow$ establishing hypothesis $\rightarrow$ hypothesis verification $\rightarrow$ making conclusion $\rightarrow$ transforming into ability. What's more, it analyzes means necessary for economic mathematics teachers to develop advanced mathematical thinking and skills for students so as to achieve the purpose of transforming freshman's primary mathematics thinking mode into higher mathematics thinking mode finally.
\end{abstract}

Keywords: Discovery teaching method; Density function; Frequency density histogram; Definite integral.

\section{Introduction}

Jerome Seymour Bruner (1915.10-2016.6), an educational psychologist and cognitive psychologist, is one of the main representatives of contemporary cognitive psychology. His learning theory is based on students' knowledge learning. Bruner's theory of learning is also called cognition and discovery. "Discovery learning" advocated by Brunner is also known as "exploratory learning", which refers to the method of cultivating inquiry thinking methods and using basic textbooks as content to enable students to learn through "rediscovery". Teaching by the discovery method is called "discovery teaching". Wang Zhu (2002) [1] introduces and evaluates discovery teaching method from three aspects, namely implementation requirements, recognized advantages and performance considered as disadvantages.

Basic characteristics of discovery teaching are as follows: a. In terms of "method", "discovery teaching" is conducted through steps (or procedures) of rediscovery. b. In terms of "content", "discovery teaching" is in principle a learning method based on teaching materials, and does not require discovery of knowledge other than teaching materials and not discovered by predecessors. $\mathrm{c}$ in terms of "objective" point of view, "discovery teaching" is a study to cultivate ability of inquiry thinking. the teaching mode of "discovery teaching" is based on cognitive structure of psychology and intuitive thinking theory.

In Bruner's view, the best state of learning is thinking, which is a process by which a person can understand a lot of messy facts perceived through activities called conceptualization or typology. Bruner demonstrated such importance of mastering structure as follows: firstly, "understanding basic principles makes subjects easier to understand." Secondly: "mastering the basic principles can ensure that memory will not be lost completely." Thirdly: "Understanding basic principles and concepts is the humanity leading to appropriate training and transfer". Fourthly: "emphasizing structure in teaching can narrow the gap between advanced knowledge and primary knowledge." Therefore, the basic operating procedures of the "discovery teaching method" are: creating a situation for finding problems $\rightarrow$ establishing hypotheses for solving problems $\rightarrow$ repeatedly verifying hypotheses $\rightarrow$ making scientific conclusions $\rightarrow$ transforming into abilities. Hu Sheng (2003) [2] Reconsidered "Discovery Teaching Method" in the teaching of physical education major in normal universities as well as found out a more suitable teaching mode for students to learn. The first level of discovery of traditional discovery teaching method is developed. It not only studies how students conduct discovery learning when learning new knowledge, but also pays more attention to in-depth discovery, 
that is, on the basis of mastering new knowledge, thinking about how to transfer the learned knowledge to the second level of discovery of others.

Xiao Jun (2012) [3] proposed a new idea of discovery teaching in the teaching of mean value theorem, and made a useful discussion on the teaching reform of higher mathematics.

Zhou Qiyuan (2014) [4] implemented discovery teaching method in the teaching of mathematical analysis course is of great significance to stimulate students' interest in learning and awareness of autonomous learning, and to cultivate students' ability to solve problems and team cooperation.

Fan Jiqiu (2016) [5] applied the discovery teaching method in extreme value teaching, so that students can consciously and actively discover the exploration knowledge and get the methods and steps to solve the problem.

Based on the ideas and viewpoints of discovery teaching method, Qiao Ximin (2016) [6] used the interesting material of linear algebra development history, created teaching situation, comprehended the process of discovering learning and discovered teaching, understood the essence of discovery teaching method in linear algebra teaching process is: Clarifying teaching objectives, strengthening the natural nature of knowledge generation, discussing the criticality of questioning reflection, correct and test conclusions, summarizing their laws in different categories, returning to the verification and application of practical problems, and comprehensively enhancing students' mathematical literacy and rational spirit.

\section{Design and Exploration}

\subsection{Creating Situation}

Probability theory is divided into discrete random variable probability and continuous random variable probability. Obviously, the density function is the key to understand the probability of continuous random variables.

Now measure the weight of 100 adult men and get 100 weight data, as shown in Table 1.

Table 1. Weight scales for 100 adult men

\begin{tabular}{cccccccccc}
\hline 60 & 60.5 & 80 & 77 & 64.5 & 59 & 51 & 43 & 46 & 61 \\
\hline 80.5 & 83 & 49 & 50 & 52 & 70 & 71 & 62 & 62 & $\mathbf{4 0}$ \\
47.5 & 71.5 & 85 & 86 & 42 & 49 & 63 & 64 & 65 & 72 \\
49.5 & 65.5 & 48 & 48 & 50.5 & 66 & 67 & 73 & 87 & 87.5 \\
\hline 88 & 68 & 68.5 & 53 & 59 & 73.5 & 69 & 56 & 54.5 & 49 \\
49.5 & 74 & 75 & 90 & 91 & 78 & 76 & 47 & 59.5 & 57.5 \\
69.5 & 69 & 45 & 50 & 57 & 63 & 64 & 79 & 79.5 & $\mathbf{9 9 . 5}$ \\
90 & 74.5 & 58 & 58.5 & 66 & 65 & 76 & 77 & 94 & 75 \\
60 & 61 & 62.5 & 48.5 & 63 & 67 & 76 & 75 & 74.5 & 97 \\
64.5 & 67.5 & 68.5 & 65 & 59 & 53 & 58 & 89 & 56 & 54 \\
\hline
\end{tabular}

Let $X$ be the weight of any adult man, $\max X=99.5, \min X=40$, which can be considered as $X \in$ $40,100)$. The interval is divided into five cells connected end to end. 
Table 2. Weight grouping table of 100 adult men

\begin{tabular}{ccccc}
\hline Grouping & Interval & Frequency (number of people) & frequency & Frequency density \\
\hline $\mathbf{1}$ (very thin) & {$[40,50)$} & 15 & 0.15 & 0.015 \\
$\mathbf{2}$ (thin) & {$[50,60)$} & 20 & 0.20 & 0.020 \\
$\mathbf{3}$ (standard) & {$[60,70)$} & 30 & 0.30 & 0.030 \\
\hline $\mathbf{4}$ (fat) & {$[70,80)$} & 20 & 0.20 & 0.020 \\
\hline $\mathbf{5}$ (very fat) & {$[80,100)$} & 15 & 0.15 & 0.0075 \\
\hline
\end{tabular}

Frequency density calculation formula:

Frequency density $=$ frequency $/$ group distance

Frequency calculation formula:

Frequency $=$ group distance $\times$ frequency density $=$ area of the corresponding rectangle

\subsection{Hypothesis}

Hypothesis If the above test for measuring body weight is done infinitely many times, there is an infinite number of data.
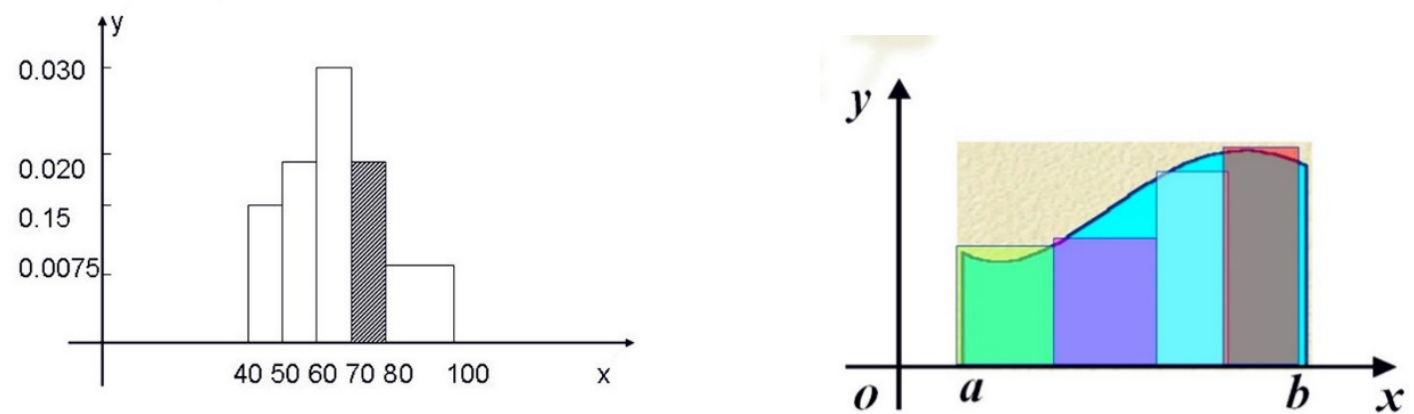

Fig 1. Frequency density histogram Fig 2 . There are 4 sets of frequency density histogram

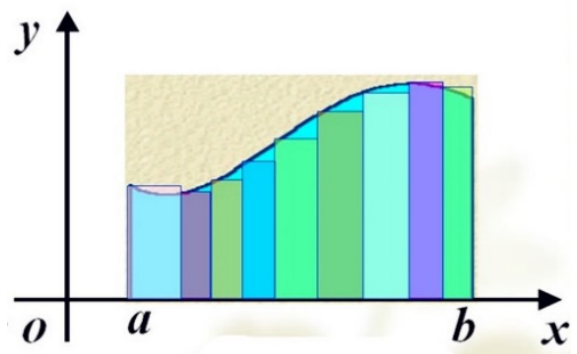

Fig 3. There are 9 sets of frequency density histograms. 


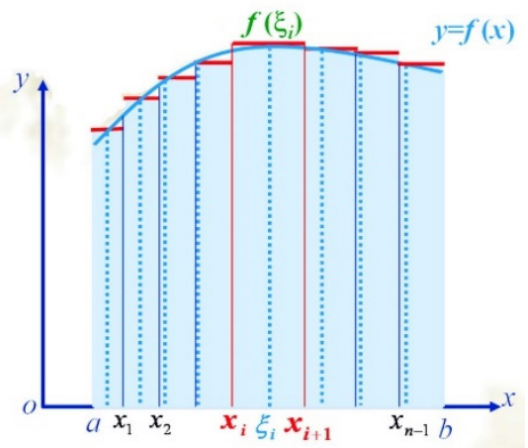

Fig 4. There are $\mathrm{n}$ sets of frequency density histograms.

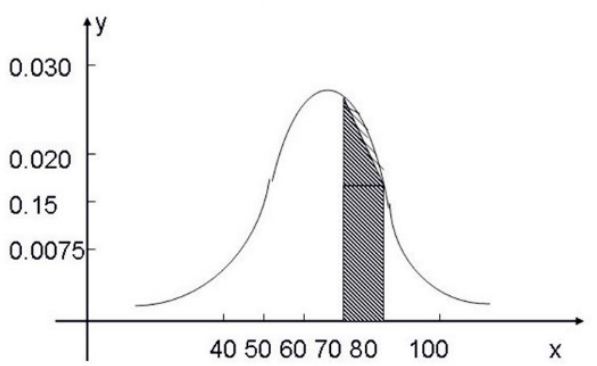

Fig 5. Curved trapezoidal area map

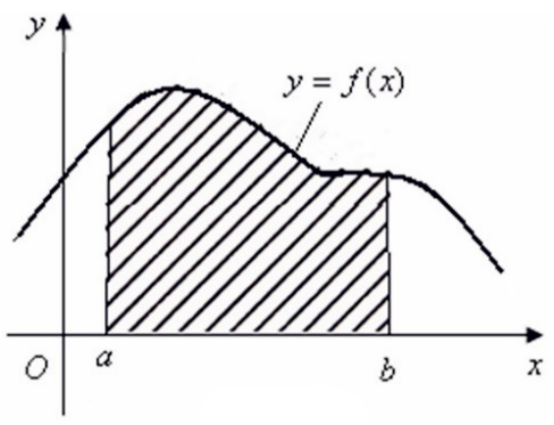

Fig 6. Curved trapezoidal area map

It can be seen from Figure 1 - Figure 4 that the group distance is small enough when grouping, and the length of the straight line parallel to the $\mathrm{x}$ axis in the histogram is close to $0 \mathrm{X}$. The frequency of the value in a certain interval is infinitely close to the probability that it takes value in this interval. Then, the probability density curve is obtained.

As can be seen from Figure 5

$$
\text { Shadow area }=P\{70 \leq X<80\}=\int_{70}^{80} \varphi(x) d x
$$

\subsection{Verification}

From a geometric point of view, the probability $P(a<\xi<b)$ is exactly the curved trapezoidal area with the probability density curve $y=f(x)$ as the top of the interval $(a, b)$. The geometric meaning of the integral of Figure 6 shows:

The curved side trapezoidal area is the algebraic sum of the area of each part enclosed by the figure $x$ axis, function $f(x)$ and the two straight lines $x=a, x=b$. The area above $x$ axis is taken as a positive sign; the area below $x$ axis is taken as a negative sign.

\subsection{Conclusion}

Since the probability of any random variable must be greater than or equal to 0 and less than or equal to 1 , the probability density function has the following properties:

(a) $f(x) \geq 0(-\infty<x<+\infty)$;

(b) $\int_{-\infty}^{+\infty} f(x) d x=P(-\infty<x<+\infty)=P(\Omega)=1$

(c) $P(\xi=a)=\int_{a}^{a} f(x) d x=0$ 


\section{Conclusion}

Taking Bruner's discovery teaching method and definition of density function as examples, whether the learning of a new mathematical concept should be directly defined by teachers or elements of exploring a new concept by using students' existing knowledge and experience are discussed in this paper. Based on five-step approach to discovery teaching: creating situation $\rightarrow$ establishing hypothesis $\rightarrow$ hypothesis verification $\rightarrow$ making conclusion $\rightarrow$ transforming into ability, new concepts are defined subsequently. Teachers can't just teach students the high-level mathematical thinking, but more importantly, let students get the process of higher mathematical thinking. The importance of university mathematics concept learning in the teaching process is the way for college students to acquire high level math skills, which is crucial for the freshman of the undergraduate business school to turn to the abstract axiomatized system thinking.

\section{Acknowledgements}

This research work was supported by Guangdong Higher Education Teaching Revolution Project: Experiencing Teaching and Mode Design of Cultivation of Internet Finance Talents under the Background of Financial Science Technology, project number: 2018SJJXGG01; Guangzhou College of Commerce Higher Education Teaching Revolution Project: Teaching Reform and Practice of Securities Investment under the Cultivation Method of Applied Talents, project number: 2018XJJXGG01. Foundation projects: Guangdong Undergraduate Higher Education Teaching Quality Engineering project: "Guangzhou College of Commerce - Applied Training Base of Mobile Commerce and E-commerce Logistics, project number: SJJD201501; Guangdong College Characteristic Innovative Project (humanities and social sciences category): "Virtual Reality Experimental Teaching Center of Guangzhou College of Commerce", project number: 2015 WTSC X114.

\section{References}

[1]. Wang Zhu, Xia Huan, Re-recognition of Bruner's discovery of teaching methods. Educator. Vol. 01 (2002) No. 1, p. 10-11.

[2]. Hu Sheng, Re-recognition of discovery teaching method. Journal of Shenyang University (Social Science). Vol. 5 (2003) No. 1, p. 115-117.

[3]. Xiao Jun, The Application of Discovery Teaching Method in the Teaching of Mean Value Theorem. Journal of Higher Correspondence Education (Natural Sciences). Vol. 25 (2012) No. 2, p. 19-20.

[4]. Zhou Qiyuan, Xiang Xuyan, Zou Qingyun, Exploration and Practice of the Discovery Teaching Method in Mathematics Analysis Course. The Guide of Science \& Education. Vol. 3 (2014) No. 2, p. 144-145.

[5]. Fan Jiqiu, Discovering the Application of Teaching Method in Extreme Value Teaching. Course Education Research. Vol. 8 (2016) No. 2, p. 143-144.

[6]. Qiao Ximin, Implementation strategy of Discovery method in the linear algebra teaching. Journal of Guiyang University (Natural Sciences). Vol.11 (2016) No. 2, p. 62-65. 